\title{
Sensorless V-type Vector-controlled IM Drive with Inherent Flux-Weakening Capability
}

\author{
S.Bolognani*, A.Faggion*, L.Peretti†, M.Zigliotto $\dagger$ \\ *University of Padova, Dept. of Electrical Engineering, Padova, Italy - silverio.bolognani@unipd.it \\ $\uparrow$ University of Padova, Dept. of Technique and Management of Industrial Systems, Vicenza, Italy - mauro.zigliotto@unipd.it
}

Keywords: Vector control, Induction motor, Sensorless drives, Flux-weakening.

\begin{abstract}
The paper deals with a voltage-fed (V-type) vector-controlled induction motor drive without speed or position sensors. At first, the drive algorithm is briefly described. Details are then given about the capability of the proposed drive to operate in the flux-weakening region with full inverter output voltage. The parameter sensitivity is also discussed and experimental results are given to validate the proposed control scheme.
\end{abstract}

\section{Introduction}

Industrial and commercial applications of induction motor (IM) drives are highly diffused. Primitive drives were normally based on scalar Volt/Hertz (V/Hz) control strategies by which the amplitude of the voltage applied to the motor is coordinated with the motor frequency. These lowperformance drives belong to the class of the voltage-fed (Vtype) drives as the control manages directly the amplitude of the voltages supplied to the motor without a closed loop current control. They are preferably offered, without speed or position sensors (sensorless drives) [10].

In the 70s, current-fed (I-type) vector-controlled IM drives start to diffuse. They control in a closed loop manner amplitude and angle of the stator current space vector, with significant advantages in terms of overall drive performance. However they involves a more complicated and costly control system. Field Oriented Control (FOC) principle gives the theory for developing such drives [9].

The great attention to costs stimulated the search of an intermediate class of drives, with a control complexity not much higher than simple V-type $\mathrm{V} / \mathrm{Hz}$ control, but performance not much lower than modern I-type FOC drives. These medium-performance drive generally are of the V-type class but they preserve the vector control features since both the amplitude and the phase (or the components in a two axis reference frame) of the stator voltage are used for the control [2]-[8], [13].

This paper proposes a sensorless V-type vector-controlled induction motor drive, able to operate in the flux-weakening region with full output voltage of the inverter. The scheme is at first described and design hints are given in the paper for the stability and tuning. The drive presents some rather substantial improvements in a previous scheme [1], which make it suitable for a broader application range. Experimental results confirm the validity of the proposed scheme.

\section{Proposed control scheme}

The block schematic of the proposed V-type sensorless drive is reported in Fig.1.

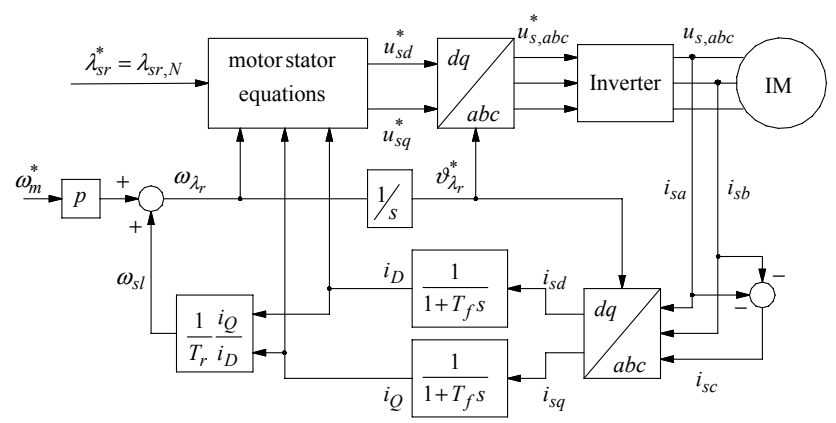

Fig. 1 - The proposed V-Type vector controlled sensorless IM drive

For its description, the current-fed (I-type) vector-controlled drive of Fig. 2 can be taken as reference. It is a quite conventional field oriented control with calculation of the slip angular frequency from the stator currents. It incorporates: (i) a closed loop speed control, the PID of which outputs the torque-producing current reference $i^{*}{ }_{s q}$, (ii) current control loops for delivering the voltage references $u^{*}{ }_{s d}$ and $u^{*}{ }_{s d}$ and (iii) flux weakening features (not explicitly shown in the figure) that correct the flux-producing current reference $i{ }^{*}{ }_{s d}$, otherwise set to its nominal level, as the speed exceeds its base value.

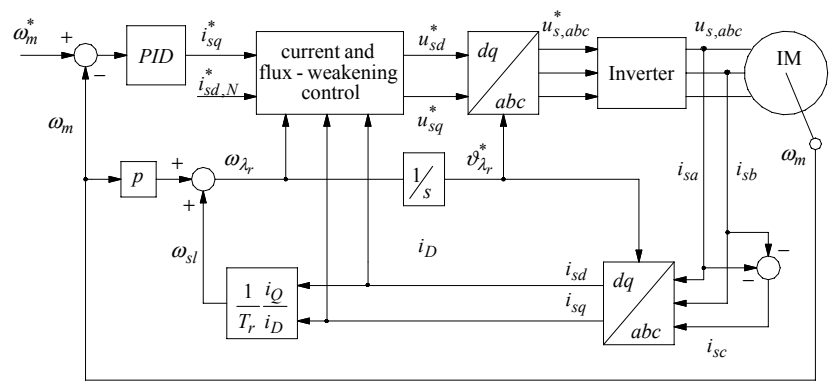

Fig. 2 - An I-Type vector controlled (FOC) sensored IM drive

In order to migrate from Fig. 2 to Fig. 1, as a first step, the current loops are substituted by the $I M$ stator voltage equations, written in a synchronous reference frame with the $\mathrm{d}$-axis fixed along to the rotor flux space vector. 


$$
\left\{\begin{array}{l}
u_{s d}^{*}=\left(R_{s} i_{s d}+L_{t} \frac{d i_{s d}}{d t}-\omega_{\lambda_{r}} L_{t} i_{s q}\right)+\frac{d \lambda_{s r}}{d t} \\
u_{s q}^{*}=\left(R_{s} i_{s q}+L_{t} \frac{d i_{s q}}{d t}+\omega_{\lambda_{r}} L_{t} i_{s d}\right)+\omega_{\lambda_{r}} \lambda_{s r}
\end{array}\right.
$$

In Equations (1), $R_{S}$ is the stator phase resistance, $L_{t}$ is the transient inductance and $\Lambda_{s r}$ is the rotor flux linkage referred to the stator, respectively. It is assumed that all the motor parameters are known.

Equations (1) deliver the voltage references as a function of the motor currents and rotor flux linkage. Motor currents are measured, while the rotor flux linkage is assumed equal to its nominal value which is constant (flux weakening action will be discussed later; anyway, rotor flux is varying only slowly with the speed under flux weakening operation). Therefore Equations (1) can be modified in the simplified form given by

$$
\left\{\begin{array}{l}
u_{s d}^{*} \cong\left(R_{s} i_{s d}+L_{t} \frac{d i_{s d}}{d t}-\omega_{\lambda_{r}} L_{t} i_{s q}\right) \\
u_{s q}^{*} \cong\left(R_{s} i_{s q}+L_{t} \frac{d i_{s q}}{d t}+\omega_{\lambda_{r}} L_{t} i_{s d}\right)+\omega_{\lambda_{r}} \lambda_{s r, N}
\end{array}\right.
$$

To make easier the implementation, the current derivatives (which are null at steady state) can also be omitted or replaced by filtered derivatives when the drive is designed for a limited dynamics.

Equations (2) point out that the required stator voltages have two contributions: the first one (within bracket in Equations (2)) are related to the stator voltage drop due to stator resistance and transient inductance: voltage drop compensation; the second one is the stator back electromotive force (bemf) due to rotor flux linkage and proportional to the rotational speed $\omega_{\lambda r}$ of the flux vector, i.e. the angular frequency of stator voltages and currents at steady-state.

It is worth noticing that, apart from very low speed operation, the bemf is the dominant term of the voltage and can be easily programmed on the basis of the rated data of the motor. The rotor flux $\lambda_{s r, N}$ can be set in order to produce the rated voltage at the base speed under no load operation.

Stator voltage drop is a secondary and minor contribution. The effects of the differences between the actual values of the motor parameters and those used in the control may thus be generally tolerated by the drive. Parameter sensitivity at low speed will be discussed later.

In addition, a low accuracy measure of stator currents can be accepted as the latter appear only in the compensation of the voltage drop. This makes the proposed drive suitable for a low cost measurement of the stator current by installing a single sensor on the DC bus of the inverter. On the contrary the current sensing has to be very precise in the current-fed FOC drive of Fig. 2. The next step is the elimination of the speed measurement, which is expensive and invalidates the mechanical robustness of the induction motor. To this aim, the actual speed in Fig. 2 can be replaced by the given speed reference. In principle, this is valid if the reference dynamics falls within the system bandwidth. Otherwise, the approximation can be made possible by the insertion of a ramp limiter block for the reference speed. The PID speed regulator in Fig. 2 can then be removed as the quadrature current reference is no more required, being only a current measurement still necessary, as shown in Fig. 1.

It is worth to note that the scheme of Fig. 1 preserves its vector control features since both the stator voltage components $u_{s d}, u_{s q}$ are used for the control. On the contrary, a strong simplification is introduced in scalar control where only the amplitude of the voltage vector is managed with loss of drive performance particularly at medium-low speed range. The proposed control scheme, indeed, manages correctly the voltage vector in all the working conditions assuring superior performance of the drive.

The lower part of Fig.1 maintains the slip calculator of the FOC schemes. Slip angular frequency is added to speed reference to generate the stator angular frequency imposed to the motor. In this way the motor speed drop due to the load torque is cancelled: slip compensation. For a good performance of the drive, the speed reference variations have to be sufficiently low to fall within the system bandwidth. To this aim, an adequate rate limiter could be profitably applied to the speed reference itself. The direct and quadrature currents used in the voltage drop compensation and in the slip compensation are really the quantities $i_{D}$ and $i_{Q}$ obtained by filtering the measured quantities with two identical low pass filters with time constant $T_{f}$. This is dictated by the drive stability requirements as illustrated in the next Section.

In the drive previously proposed in [1], the $\mathrm{i}_{D}$ current is derived from the reference of the rotor flux referred to the stator, $\lambda_{s r, N}$, while here it is obtained by filtering the measured current $i_{s d}$. This makes the control system more symmetrical, and it smoothes the way for the automatic flux-weakening control, which is the latest innovation. The original version of the drive instead exhibits its best in the constant flux region. Of course, the two control schemes can be easily merged to use the best one for each speed range.

\section{Stability analysis}

A delicate issue of the proposed scheme is the verification of its stability, as instability can be suspected because the control uses the measured currents to define the motor voltages which produce the currents that are measured.

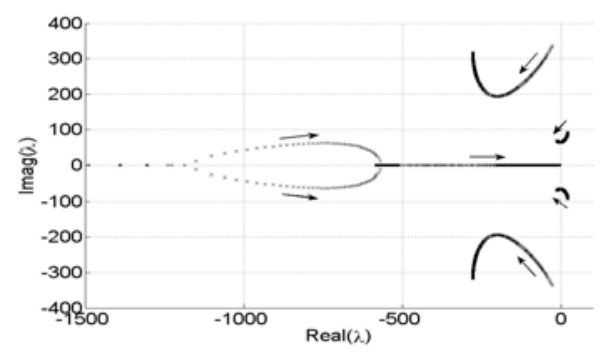

Fig. 3 - Root locus of the drive, for different values of the current filters time constant (arrows indicate increasing values)

The stability analysis has been faced by computing the root locus of the system (mechanical load included) linearised around an operating point. It can be demonstrated that the system stability is affected by a proper choice of the time 
constant $T_{f}$ of the low pass filters on the $i_{s d}, i_{s q}$ currents. Fig. 3 reports the root locus of the linearised system of Fig. 1 around a selected working point (about $50 \%$ of rated torque and $30 \%$ of rated speed), for increasing $T_{f}$ starting from a null value. Clearly, the stability is guaranteed only with $T_{f}$ higher than a threshold, which increases with the inertia of the driven mechanical system. An appropriate time constant can be designed to meet all the working conditions. It is of the order of some tens of milliseconds for drives of some kilowatts or less of rated power.

\section{Flux-weakening operation}

The most interesting add-on feature of the proposed drive is its inherent flux-weakening capability. It is obtained by a proper limitation of the inverter voltage references, which derive from the motor equations. When the voltage inverter saturates, the actual rotor flux $\lambda_{s r}$ in the motor falls off below the nominal value $\lambda_{s r, N}$, which is used in the motor stator equations block of Fig.1. Many known schemes act in some open or closed-loop form to reduce the flux reference as the control approaches the inverter limits (flux-weakening). Conversely, when the reference voltage computed by Equations (2) exceeds the inverter limits, the proposed V-type control applies a limitation to quadrature voltage only, while maintaining the same flux reference. From Equations (2), one realizes that a automatic limitation of $u_{s q} *$ is equivalent to a reduction of rotor flux reference, exactly of the amount needed to maintain the produced voltage at inverter's limits. According to the space vector modulation, a given reference voltage space vector is obtained as the time-weighted average of two adjacent state vectors in the stationary reference frame. The period of application of each vector can be also expressed in the selected $d-q$ frame, synchronous to the rotor flux, as follows:

$$
\begin{aligned}
T_{m} & =\sqrt{3} \frac{T_{c}}{U_{d c}}\left[u_{d} \sin \left(m \frac{\pi}{3}-\theta_{d q}\right)-u_{q} \cos \left(m \frac{\pi}{3}-\theta_{d q}\right)\right] \\
T_{m+1} & =\sqrt{3} \frac{T_{c}}{U_{d c}}\left[u_{d} \sin \left(\theta_{d q}-(m-1) \frac{\pi}{3}\right)+u_{q} \cos \left(\theta_{d q}-(m-1) \frac{\pi}{3}\right)\right]
\end{aligned}
$$

where $\mathrm{m}=1, \ldots, 6$ is the sector index of the hexagonal feasible inverter voltage region in the stationary $\alpha-\beta$ plane, $T_{c}$ is the modulation period, $U_{D C}$ is the inverter DC bus voltage, and $\theta_{d q}$ is the angular position of the synchronous rotating reference frame wich is equal to $\theta^{*} \lambda r$ in Fig. 1.

The proposed voltage limitation strategy consists in preserving the $d$-axis component of the demanded voltage, while constraining the $q$-axis voltage as required by the hexagonal boundary. This is quite easily obtained by acting upon the $u_{q}$-dependant terms of both $T_{m}$ and $T_{m+1}$. This asymmetrical limitation of the reference voltage yield a straightforward flux-weakening action, as shown by the experimental results of Fig.4, for a motor with base speed of $3000 \mathrm{rpm}$. It is evident the reduction of the $d$-axis (fluxproducing) component of the stator current, tied up the $q$-axis voltage clamp. Under no load operation, the torque-producing current $i_{s q}$ is approximately null.
When driving a constant load torque, flux-weakening enables a further increase of the torque-producing component, to get a constant electromagnetic torque delivery. Operation at maximum speed and $75 \%$ of rated torque as in Fig. 4 can be tolerated only for a short time as the current level exceed the rated value of $4 \mathrm{~A}$.
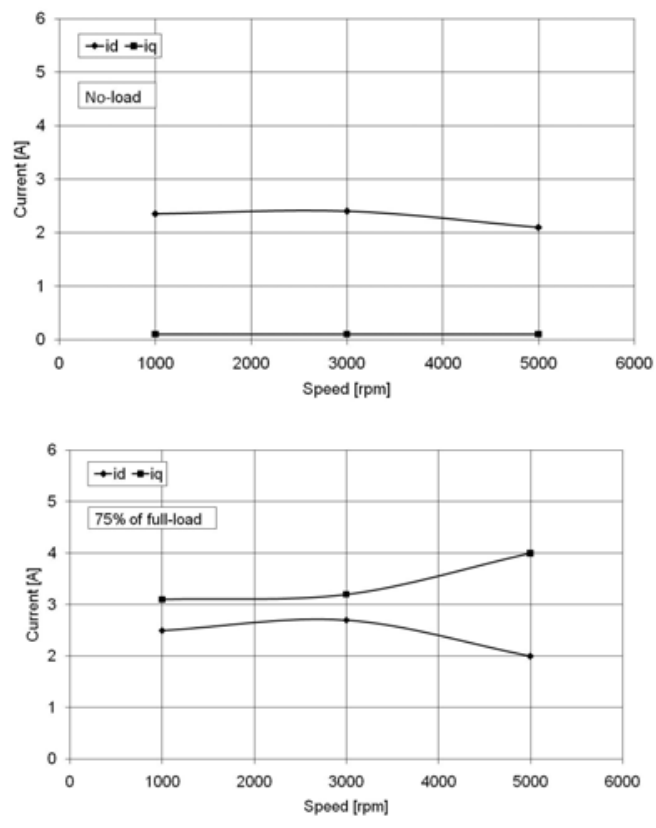

Fig. 4 - Currents during automatic flux-weakening - no-load (top) and 75\% of rated torque (bottom).

\section{Experimental tests}

An extensive set of performance tests has been carried out. An example of experimental results is reported in Fig. 5. The figure refers to a constant speed reference of $500 \mathrm{rpm}(1 / 6$ of the base speed or $1 / 10$ of the maximum speed), with increasing load torque from zero (no load operation) to full load given by $1.4 \mathrm{Nm}$ and $4 \mathrm{~A}$ as amplitude of the current vector (2.8 Arms phase current). Torque is measured by a torquemeter incorporated in the hysteresis brake used as mechanical load. One can note a constant speed operation with the torque, slightly higher than the reference. The speed error is lower than $0.3 \%$ of the base speed.

Flux-producing current $i_{s d}$ remains constant too (assuring constant flux operation) while the torque producing component $i_{s q}$ increases quite linearly with the torque

The lower curve in the figure compares the measured torque with the estimated torque $\mathrm{m}_{e}$ generated by the motor computed by

$m_{e}=\frac{3}{2} p \frac{L_{M}^{2}}{L_{r}} i_{s d}^{x} i_{s q}^{x}=\frac{3}{2} p L_{\varphi} i_{s d}^{x} i_{s q}^{x}$

where $\mathrm{p}$ is number of the pole pairs and $L_{M}$ and $L_{r}$ are the mutual inductance between stator and rotor d- (or q-) windings and the rotor inductance respectively and $L_{\varphi}$ is the ratio $\lambda_{s r} / i_{s d}$ evaluable from plate data of the motor. A good correspondence between measured and estimated torque can 
be observed. The small discrepancy can be due to mechanical losses of the motor and light erroneous values of the parameters used in the control. The test has been repeated by reducing to $70 \%$ the stator resistance value implemented into the control scheme, to emulate an increase of the motor resistance due to a temperature rise. The new torque-speed characteristic is shown by a dashed curve in Fig. 5. The speed error is larger and increasing with the torque up to $2 \%$ of base speed at rated torque. An analytical justification of the increment is given in the last Section of the paper.
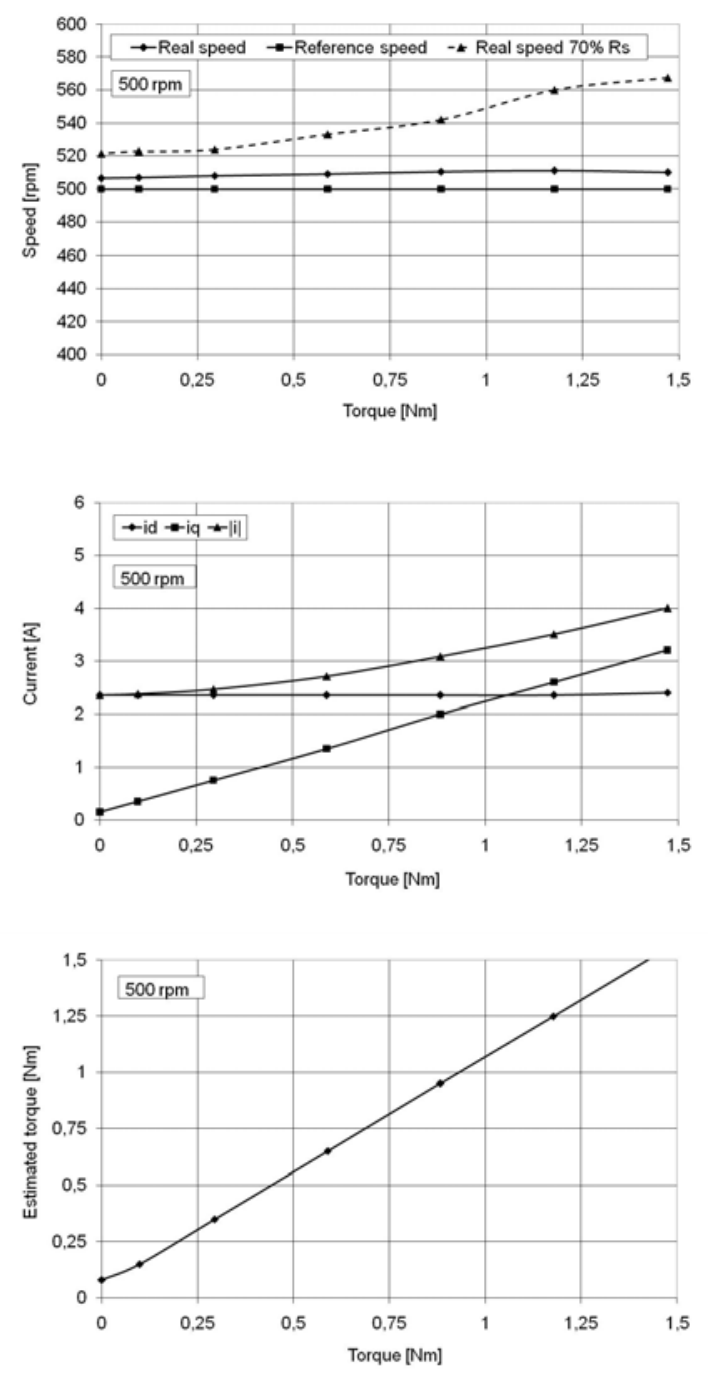

Fig. 5 - Drive performance at $500 \mathrm{rpm}$ - speed (top), currents (middle), estimated torque (bottom) as functions of the actual torque

Another example of experimental results is reported in Fig. 6 , carried out at a constant speed reference of $5000 \mathrm{rpm}$ which is the maximum flux-weakening speed of the drive $(5 / 3$ of the base speed). The torque is increased from zero to $1.2 \mathrm{Nm}$ that is higher than the continuous torque limit at that speed. This can be realized by observing that the stator current is higher than the rated value of $4 \mathrm{~A}$. One can note again a quite constant speed operation. The maximum speed error occurs at the maximum torque and it is equal to $-0.7 \%$ of the base speed. Flux-producing current $i_{s d}$ remains approximately constant at a level lower than that of Fig. 5, confirming the flux weakening intervention. The test is now repeated decreasing to $70 \%$ the stator resistance value implemented into the control scheme. The operating speed is still higher than without resistance reduction as shown by the dashed curve in the upper part of Fig. 6, but the speed error remains very limited.
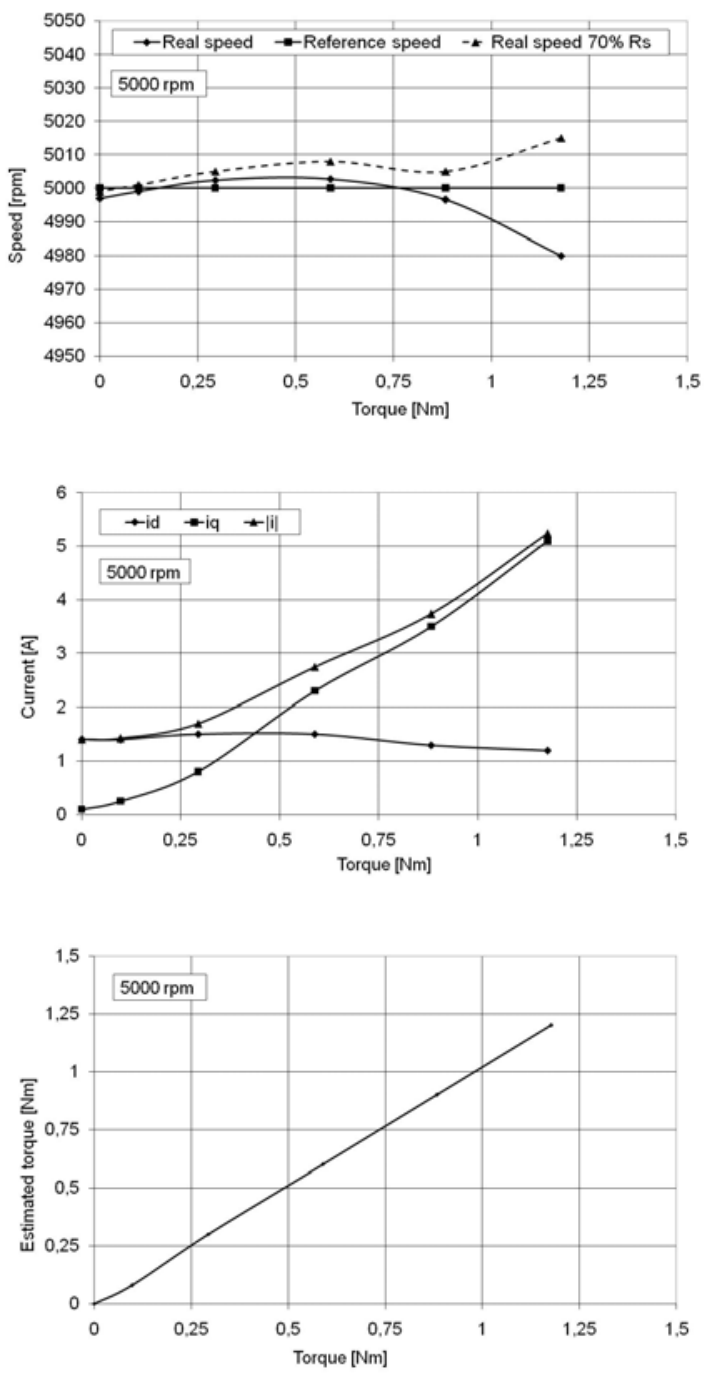

Fig. 6 - Drive performance at $5000 \mathrm{rpm}$ - speed (top), currents (middle), estimated torque (bottom) as functions of the actual torque

\section{Parameter sensitivity}

One can note that only two parameters are involved in the motor equations used to define the stator voltage references in the control scheme, i.e. $R_{s}$ and $L_{t}$. Every time parameter values not coincident to the actual ones are used, wrong voltages are applied to the motor, causing wrong steady state speed with respect to the speed reference. The amount of steady state speed error for given parameter error is an index of the parameter sensitivity of the drive control. In addition to the speed error, parameter mismatch causes also variation in the stator current and error in the estimation of the rotor flux vector angle, that is $d-q$ reference frame position. A simple evaluation of the sensitivity can be performed under no-load 
operation of the motor. In such a condition, the rotor currents are null (or negligible in practical no-load) and then the steady state stator equations in a generic d-q reference frame rotating synchronously with any vectors of the stator quantities are

$$
\left\{\begin{array}{l}
u_{s d}^{x}=R_{s} i_{s d}^{x}-\omega_{x} L_{s} i_{s q}^{x} \\
u_{s q}^{x}=R_{s} i_{s q}^{x}+\omega_{x} L_{s} i_{s d}^{x}
\end{array}\right.
$$

while the same voltages imposed by the control are

$$
\left\{\begin{array}{l}
u_{s d}^{*}=R_{s}^{\prime} i_{s d}^{x}-\omega_{x} L_{t}^{\prime} i_{s q}^{x} \\
u_{s q}^{*}=R_{s}^{\prime} i_{s q}^{x}+\omega_{x} L_{t}^{\prime} i_{s d}^{x}+\omega_{x} \lambda_{s r, N}
\end{array}\right.
$$

where $R_{s}^{\prime}$ and $L_{t}^{\prime}$ are the presumed parameters and $\omega_{x}$ is the rotational speed of the reference frame, that corresponds to the speed of the motor for the assumption of no load operation. Parameter $L_{s}$ is the stator inductance. By equating the voltages in Equations (5) and (6) and solving for the stator currents one obtains

$$
\left\{\begin{array}{l}
i_{s d}^{x}=\frac{\omega_{x}^{2} \lambda_{s r, N}\left(L_{s}-L_{t}^{\prime}\right)}{\left(R_{s}-R_{s}^{\prime}\right)^{2}+\omega_{x}^{2}\left(L_{s}-L_{t}^{\prime}\right)^{2}} \\
i_{s q}^{x}=\frac{\left(R-R_{s}^{\prime}\right) \omega_{x} \lambda_{s r, N}}{\left(R_{s}-R_{s}^{\prime}\right)^{2}+\omega_{x}^{2}\left(L_{s}-L_{t}^{\prime}\right)^{2}}
\end{array}\right.
$$

From Equations (7), the slip angular frequency results

$$
\omega_{s l}=\frac{1}{T_{r}} \frac{i_{s q}^{x}}{i_{s d}^{x}}=\frac{1}{T_{r}} \frac{\left(R_{s}-R_{s}^{\prime}\right)}{\omega_{x}\left(L_{s}-L_{t}^{\prime}\right)}
$$

Equations (7) and (8) point out the influence of parameter error to no-load drive performance. In particular from Equations (7) one can realize that in presence of a stator resistance mismatch, a quadrature current different from zero occurs in the motor windings provided that the angular speed of the $d-q$ reference frame is different from zero. In addition, from Equation (8), it results that the slip angular frequency, that corresponds to the speed error, is zero only if the correct value of the stator resistance is used in the control. This interesting results can be exploited to tune the voltage equations implemented in the V-type drive scheme of Fig. 1, by choosing the value that nullify the angular slip under no load operation at speed different from zero. This approach for the stator resistance tuning has been used for the test reported in

Fig. 7 at $125 \mathrm{rpm}$ equal to $4 \%$ of the base speed of the drive (or only $2.5 \%$ of the maximum speed). After the no load measurement and tuning, the load torque has been increased and the torque-speed characteristic of the figure has been measured, confirming the good performance of the drive. Of course, if no load operation is not allowed, the stator resistance estimation can be performed by one of the method presented in [12]. As far as $L_{t}$ is concerned, its sensitivity on drive speed accuracy is generally lower than that of stator resistance, since the value of $L_{t}$ is compared to $L_{s}$ that is much higher.

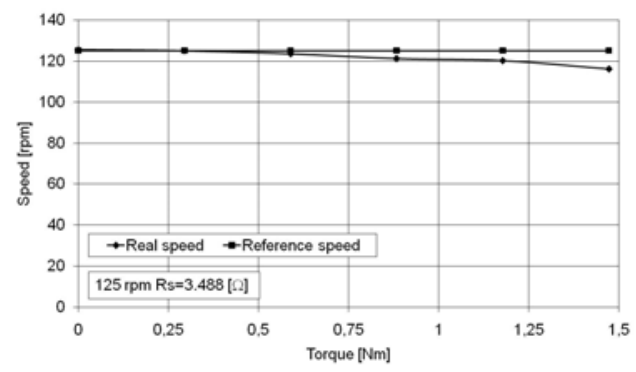

Fig. 7 Torque-speed characteristic at $125 \mathrm{rpm}$

\section{Acknowledgements}

The authors greatly thank Ing. Ivan Tocca for his valuable contribution during the experimental stage of the work.

\section{References}

[1] S.Bolognani, L.Tubiana, M.Zigliotto, "V-Type Sensorless Field Oriented Control of Induction Motors for Low Cost Applications", EPE-PEMC'04, Riga, Latvia, CD-ROM, 2004.

[2] A.M.García, T.A.Lipo, D.W.Novotny, "A New Induction Motor V/f Control Method Capable of High Performance Regulation at Low Speed", IEEE Trans. on Industry Applications, pp.813821, Vol.34, No.4, July/August 1998.

[3] Gastli, M. Tomita, T. Takeshita and N. Matsui, "Improvement of a Stator-Flux-Oriented Speed-Sensorless Control of an Induction Motor", IEEE PCC, Yokohama, pp.415-20, 1993.

[4] S.Jin, Z.Wei, H.Zhenyi, "One Novel Scalar Control Scheme for Induction Machine", IEEE Industrial Electronics Conference, IECON'04, pp.347-352, 2006.

[5] R.J.Kerkman, T.M.Rowan, "Voltage-Controlled CurrentRegulated PWM Inverters", IEEE Trans. On Industry Applications, pp.244-251, Vol.26, No.2, March/April 1990.

[6] K.Koga, R.Ueda, T.Sonoda, "Achievement of High Performances for General Purpose Inverter Drive Induction Motor Drive System", Proc. of IEEE Industry Application Society Annual Meeting, IAS'89, p.658-664, 1989.

[7] K.Koga, R.Ueda, T.Sonoda, "Constitution of V/f Control for Reducing the Steady State Speed Error to Zero in Induction Motor Drive Systems", Proc. of IEEE Industry Application Society Annual Meeting, IAS'90, pp.639-646, 1990

[8] K.Koga, R.Ueda, T.Sonoda, "Evaluation of Operating Performances of Three Typical V/f control Schemes in PWM Inverter Drive Induction Motor System", proc. of the 17th Annual Conference of the IEEE Industrial Electronics Society, IECON'91, 1991.

[9] D.W.Novotny, T.A.Lipo, "Vector Control and Dynamics of AC Drives", Clarendon Press, Oxford, 1996.

[10] A.M.Trzynadlowski, "Control of Induction Motors", ISBN 012-701510-8, Academic Press, London, 2001.

[11] P.Vas, "Parameter Estimation, Condition Monitoring, and Diagnosis of Electrical Machines", Oxford University Press, New York, ISBN 0-19-859375-9, 1993

[12] P.Vas, "Sensorless Vector and Direct Torque Control", Oxford University Press, New York, ISBN 0-19-85645-1, 1998

[13] C.C.Wang, C.H.Fang, "Sensorless scalar controlled Induction Motor drives with Modified Flux Observer" IEEE Power Engineering Review, Vol. 22, No. 8, Aug. 2002, pp. 61-68. 